\title{
Sufficient Conditions for Apparent Horizons in Spherically Symmetric Initial Data
}

\author{
Jemal Guven ${ }^{(1)} *$ and Niall Ó Murchadha ${ }^{(2) \dagger}$ \\ (1) Instituto de Ciencias Nucleares \\ Universidad Nacional Autónoma de México \\ Apdo. Postal 70-543, 04510 México, D.F., MEXICO \\ (2) Physics Department, University College Cork \\ Cork, IRELAND
}

\begin{abstract}
We establish sufficient conditions for the appearance of both apparent horizons and singularities in spherically symmetric initial data when spacetime is foliated extrinsically. Let $M$ and $P$ be respectively the total material energy and the total material current contained in some ball of radius $\ell$. Suppose that the dominant energy condition is satisfied. We show that if $M-P \geq \ell$ then the region must possess a future apparent horizon for some non -trivial closed subset of such gauges. The same inequality holds on a larger subset of gauges but with a larger constant of proportionality which depends weakly on the gauge. This work extends substantially both our joint work on moment of time symmetry initial data as well as the work of Bizon, Malec and Ó Murchadha on a maximal slice.
\end{abstract}

* jemal@nuclecu. unam.mx

$\dagger$ niall@ucc.ie 


\section{INTRODUCTION}

This paper is part of an ongoing examination of the constraints in spherically symmetric general relativity [1] 3]. Here we would like to establish sufficient conditions for the appearance of apparent horizons and singularities in general initial data. Because of their very different nature we defer the examination of necessary conditions to another publication [4].

Ideally one would like to go about this in a manifestly covariant way avoiding the necessity to introduce a gauge. Unfortunately, this is well beyond our present technical capacity. We proceed in a canonical way: the initial data consists of the intrinsic and extrinsic geometry on some spacelike hypersurface satisfying the constraints [1]

$$
K_{R}\left[K_{R}+2 K_{\mathcal{L}}\right]-\frac{1}{R^{2}}\left[2\left(R R^{\prime}\right)^{\prime}-R^{\prime 2}-1\right]=8 \pi \rho
$$

and

$$
K_{R}^{\prime}+\frac{R^{\prime}}{R}\left(K_{R}-K_{\mathcal{L}}\right)=4 \pi J
$$

We have parametrized the line element on the spatial geometry as follows

$$
d s^{2}=d \ell^{2}+R^{2} d \Omega^{2}
$$

where $\ell$ is the proper radial distance on the surface and $R$ is the areal radius. All derivatives are with respect to $\ell$. In a spherically symmetric spacetime, the extrinsic curvature is completely characterized by the two scalar functions $K_{\mathcal{L}}$ and $K_{R}$, proportional respectively to the velocities of $\ell$ and $R$ normal to the hypersurface. If $n^{a}$ is the outward pointing unit normal to the two-sphere of fixed radius in the hypersurface, we can write

$$
K_{a b}=n_{a} n_{b} K_{\mathcal{L}}+\left(g_{a b}-n_{a} n_{b}\right) K_{R}
$$

We assume that both $\rho$ and $J$ are appropriately bounded functions of $\ell$ on some compact support. We choose to foliate spacetime extrinsically. This involves fixing some scalar function of the extrinsic curvature tensor, $K_{a b}$. We focus on the subset of extrinsic time foliations of spacetime of the form

$$
K_{\mathcal{L}}+\alpha K_{R}=0
$$

where $0.5 \leq \alpha<\infty$ but is otherwise an arbitrary functional of the initial data, $R$ and $K_{R}$. $\alpha=0.5$ and $\alpha \rightarrow \infty$ define the superspace lightcone. While this might not be the most general extrinsic time gauge, a very large degree of freedom is admitted.

If cosmic censorship is valid, the existence of an apparent horizon provides a natural boundary on the configuration space between regular data and data that is singular or will develop a singularity.

We recall that a future (past) apparent horizon exists when the divergence $\Theta_{+}\left(\Theta_{-}\right)$of outward pointing, future (past) directed null rays vanishes on a closed surface - in our case a two -sphere of fixed proper radius. It is easy to show [5], for spherical initial data, that if there exists a non-spherical trapped surface or apparent horizon then there also must exist a spherical one. We can express $\Theta_{ \pm}=\omega_{ \pm} / R$, where 


$$
\omega_{ \pm}=2\left(R^{\prime} \pm R K_{R}\right),
$$

are the optical scalars introduced in [6]. A future apparent horizon therefore occurs whenever $\omega_{+}=0$. If $\omega_{+} \leq 0$, we say that the surface is future trapped. The transcription for past horizon will always be obvious so henceforth will be omitted. With $\omega_{+}$and $\omega_{-}$, we can reconstruct the lightcone at each point on the hypersurface (this depends both on the intrinsic and on the extrinsic geometry). In particular, it is often useful to cast the constraints in terms of these variables when we are interested in identifying apparent horizons [6] [1, 3].

When $K_{a b}=0$, the location of an apparent horizon, when one exists, coincides with an extremal surface of the spatial geometry [2]. In a spherically symmetric geometry these are locations where $R^{\prime}=0$. In general, apparent horizons do not coincide with extremal surfaces of the spatial geometry. Initial data with an apparent horizon need not even possess an extremal surface and, vice versa.

The spatial geometries we consider consist of a single asymptotically flat region with topology $R^{3}$. The appropriate boundary condition on the metric at the base of the geometry at $\ell=0$ is then

$$
R(0)=0 .
$$

We suppose that the center is regular so that $R^{\prime}(0)=1$ and $K_{R}(0)=0$. If no singularity intervenes between the base and infinity we will say that the geometry is regular. In this geometry, the integrated action of the interior distribution of source energy-momentum can potentially produce an apparent horizon.

To cast a sufficient condition for the existence of an apparent horizon we suppose that the spatial geometry does not possess any future trapped surfaces and is regular in some bounded region containing the origin $\left(\omega_{+}>0\right.$ there). One needs to then show that some measure of the material energy content in this region must be bounded by a measure of the volume of the region.

The challenge is to identify a useful measure of the material energy content of a region. We follow the development of a sufficiency condition by Bizon, Malec and Ó Murchadha [7,5] and more recently by Malec and Ó Murchadha [6] and [2]. At a moment of time symmetry, the natural measure of material energy for casting a sufficiency condition for an apparent horizon was shown to be the material energy, $M$. For general initial data, the corresponding measure for a future (past) apparent horizon was shown to be the difference (sum), $M \mp P$, where $P$ is the total radial material momentum given by integrating the material current over the proper spatial volume,

$$
(M, P)=4 \pi \int_{0}^{\ell} d \ell R^{2}(\rho, J) .
$$

The greater the net outward flux (positive $P$ ), the lower $M-P$ - the more difficult to form a future apparent horizon.

Clearly, we need to make some assumptions about matter to proceed. We will assume that matter satisfies the dominant energy condition in this region:

$$
|J| \leq \rho .
$$

When the dominant energy condition is satisfied, $M \pm P$ is positive. 
Let $\ell$ be the proper radius of this region. Let the dominant energy condition hold everywhere. Let $1 \leq \alpha \leq 2$ but be otherwise arbitrary. Then if

$$
M \mp P \geq \ell,
$$

the region must contain a future (past) apparent horizon. This is the central result of this paper.

The inequality (10) is particularly impressive because, when $P=0$, it coincides with the moment of time symmetry result which we know to be sharp. Unlike the moment of time symmetry scenario where one could fall back on piecewise-constant density models to guide us, no such exactly solvable safety net is available here. Even the analogue of the constant density star proves to be analytically intractable when $J \neq 0$.

Bizon, Malec and Ó Murchadha [7.5] using a maximal slice $(\alpha=2)$, had earlier demonstrated that if the weak energy condition holds and if

$$
M-P \geq \frac{7}{6} \ell,
$$

the spatial geometry must contain a future trapped surface [7.5]. The numerical coefficient appearing on the right hand side is not as good as that appearing in Eq.(10). They showed, however, that this coefficient is sharp, by explicitly constructing a solution with $M-P \geq$ $(7 / 6-\epsilon) \ell$ but without any trapped surface. This solution notably did not satisfy the dominant energy condition.

The result Eq.(10) was first established by Malec and Ó Murchadha for maximal slices [6]. These earlier results were derived assuming global regularity, a condition we do not require.

The new idea introduced in [6] was to recast the constraints of the theory in terms of the optical scalar variables [6] [1,3]. This permits one to enforce the dominant energy condition rigorously - even when $\alpha$ varies so long as it does not stray too close to the superspace lightcone.

With maximal slicing alone to go by, one can not be certain that the inequality possesses any gauge invariant significance - our measures of energy and size, after all, are not spacetime scalars so one rightly hesitated before jumping to physical conclusions. The principal strength of the inequality we have derived is that it not tied to the maximal gauges; the result is valid for all slices which remain within the band $1 \leq \alpha \leq 2$ lying at the center of the superspace lightcone. At least within the framework of the extrinsic time foliations, the result does not appear to be sensitive to a change in foliation. It is not at all obvious that such a happy outcome is possible. It is remarkable in view of the global nature of the problem that we can do this for $\alpha$ which is not constant.

What happens when we move outside this band? If we relax the gauge to $1 \leq \alpha<\infty$, it is still possible to establish an inequality of this form. However, a constant of proportionality, $C>1$, is introduced. $C$ will be finite if $\alpha$ is but diverges linearly as $\alpha \rightarrow \infty$. However, this limit corresponds to the polar gauge which breaks down at a horizon.

If we relax the gauge in the other direction to cover all apparently valid $\alpha$, so that $0.5 \leq \alpha<\infty$, such a bound no longer exists. There appears to be a genuine obstruction to casting a strong statement of sufficiency of the form described above as we approach the superspace lightcone. We need first to weaken the hypothesis to obtain an inequality. 
One way to do this, taking advantage of dominant energy, is to assume that the interior contains neither future nor past trapped surfaces. - the cost is that the inequality we obtain does not distinguish between future and past apparent horizons. When we do this, we obtain an inequality of the form (10), with some new constant of proportionality, $C>1$ which depends weakly on the gauge. In fact, it is finite even when $\alpha=0.5$ everywhere.

To track the potential mischief uncontrolled currents can play, we will relax the energy condition to the weak energy condition which places no constraint on $J$. The weak and the dominant are the two energy conditions that are relevant in initial data. The strong energy condition which bounds spatial stresses involves the dynamical Einstein equations in addition to the constraints.

With the weak energy condition, we find we do not fare so well. We again find, with the strong form of the hypothesis, that we can only prove sufficiency for $1 \leq \alpha<\infty$. However, the constant $C$ will now depend on $\alpha$ for all $\alpha>1$. It is mimimized at $\alpha=1$ where it asumes the optimal value +1 - the dominant and weak energy results coincide in this gauge.

We also will construct a sufficiency condition for a singularity in the initial data. Singular geometries can occur even though both $\rho$ and $J$ are finite. But the only way that the geometry can become singular is by pinching off at some finite proper radius from the center. In Sect.6, we demonstrate that the corresponding inequality for singularities can be cast as $M \geq 2 \ell$ which is independent of $\alpha$ and $P$.

The paper is organized as follows: In Sect. 2 we focus on the dominant energy condition. We begin, in Sect.2.1 with a derivation of bounds on the configuration variables which hold in regions with a regular center which do not possess an apparent horizon. These generalize bounds obtained in [3] for globally regular geometries. In Sect. 2.2 we derive a useful inequality which we use in Sect.2.3 to derive (10). In Sects.2.4 and 2.5, we relax the upper and lower range of $\alpha$ respectively. In Sect.3, we relax the energy condition. In Sect.4, we examine some interesting special cases. In particular, we show that outgoing null fluids cannot form future trapped surfaces. In Sect.5 we examine singularities in the initial data. 


\section{APPARENT HORIZONS: DOMINANT ENERGY}

\section{II.1 Bounds on the Configuration Space in Regions without Trapped Surfaces}

We consider some finite region containing the origin. To derive a sufficiency condition in the strong form, we assume only that this region is regular and does not possess any future trapped surfaces $\left(\omega_{+}>0\right.$ there $)$ — we make no assumptions concerning what happens to the geometry outside, it may contain trapped surfaces, it may even be singular.

Suppose that the dominant energy condition holds at every point in this region. When the dominant energy condition holds, our experience suggests that the appropriate variables to exploit are the optical scalars.

Even though the geometry is regular within this region, we cannot suppose that it remains so outside. In particular, we cannot impose the the same boundary conditions we did in [3] for everywhere regular geometries where we could set $R^{\prime} \rightarrow 1$, and $R K_{R} \rightarrow 0$ at infinity consistent with asymptotic flatness. Remarkably, it is possible to gain all the control over $\omega_{+}$and $\omega_{-}$we will need without any asymptotic control.

We can combine the two constraints to get simple equations for the spatial derivative of $\omega_{+}$and $\omega_{-}$:

$$
\begin{aligned}
& \left(\omega_{+}\right)^{\prime}=-8 \pi R(\rho-J)-\frac{1}{4 R}\left(\omega_{+} \omega_{-}-4\right)+\omega_{+} K_{\mathcal{L}}, \\
& \left(\omega_{-}\right)^{\prime}=-8 \pi R(\rho+J)+\frac{1}{4 R}\left(\omega_{+} \omega_{-}-4\right)-\omega_{-} K_{\mathcal{L}} .
\end{aligned}
$$

We can, in turn, combine Eqs.(12) and (13) to give

$$
\left(\omega_{+} \omega_{-}\right)^{\prime}=-8 \pi R\left[\left(\omega_{+}+\omega_{-}\right) \rho-\left(\omega_{+}-\omega_{-}\right) J\right]-\left(\omega_{+}+\omega_{-}\right)\left(\omega_{+} \omega_{-}-4\right) .
$$

It was shown in [6] and again in [1] that if we had initial data which satisfied the dominant energy condition and which was regular at both the origin and infinity then a consequence of Eq.(14) was

$$
\omega_{+} \omega_{-} \leq 4
$$

Let us assume instead that we have initial data which is regular at the origin, satisfies the dominant energy condition and has no future trapped surface inside some ball of radius $\ell$. Then inside this radius Eq.(15) holds. The proof is very easy. We know that $\omega_{+} \omega_{-}=4$ at the origin and that it decreases as soon as dominant matter is entered. Let us assume that it rises up again to 4 . However, since $\omega_{+}$is positive there so also must $\omega_{-}$be positive. Then the dominant energy condition and Eq.(14) gives us that $\left(\omega_{+} \omega_{-}\right)^{\prime} \leq 0$ there, a contradiction. This result is gauge invariant.

An even better result holds if $1 \leq \alpha<\infty$ in the finite region we consider. In [3] it was shown that when $\alpha \geq 1$, if we had globally regular maximal initial data which satisfied the dominant energy condition then

$$
-2 \leq \omega_{+}, \omega_{-} \leq 2
$$


While we need the information at infinity to set the lower bound, we can get the upper bound just from regularity at the origin. Both $\omega_{+}$and $\omega_{-}$start out equalling 2 at the origin and then decrease as one moves away. Assume that $\omega_{+}$increases up to 2 again ahead of $\omega_{-}$. However, once it reaches 2 , from the dominant energy condition and

$$
\left(\omega_{+}\right)^{\prime}=-8 \pi R(\rho-J)+\frac{1}{4 R}\left(4+(\alpha-1) \omega_{+} \omega_{-}-\alpha \omega_{+}^{2}\right)
$$

we get that $\left(\omega_{+}\right)^{\prime} \leq 0$, a contradiction. We obtain the same result for $\omega_{-}$. This does not depend on the existence of a horizon. Note that we only demand $\alpha \geq 1$, we do not require that it be a constant.

This is not, unfortunately, true if $\alpha<1$ anywhere - indeed, we constructed an explicit counterexample in [3]. If, however, the region possesses neither future nor past apparent horizons, we can prove that $\omega_{ \pm} \leq \Omega$, where

$$
\Omega=\operatorname{Max}\left(2,2 / \alpha_{\operatorname{Min}}\right) .
$$

The proof is simply a rerun of that for $\alpha \geq 1$.

Of course, if we demand global rather than just local regularity we can exploit the full range of inequalities derived in [3]. With weak energy, we have $-1 \leq R^{\prime} \leq+1$. With dominant energy, and $1 \leq \alpha<\infty$, we have

$$
\left|\omega_{ \pm}\right| \leq 2
$$

so that $R K_{R}=\left(\omega_{+}-\omega_{-}\right) / 4$ and, of course, $R^{\prime}=\left(\omega_{+}+\omega_{-}\right) / 4$ are also bounded. We have

$$
\left|R K_{R}\right| \leq 1
$$

and so also get $-1 \leq R^{\prime} \leq+1$.

\section{II.2 A Useful Inequality when $1 \leq \alpha \leq \infty$}

The optical scalar which marks the presence of a future apparent horizon is $\omega_{+}$. Its spatial gradient is determined by Eq.(17). To obtain the appropriate weighting on the sources, let us recast Eq.(17) as an equation for the spatial gradient of $R \omega_{+}$:

$$
\left(R \omega_{+}\right)^{\prime}=-8 \pi R^{2}(\rho-J)+\frac{1}{4}\left[4+\alpha \omega_{+} \omega_{-}+(1-\alpha) \omega_{+}^{2}\right] .
$$

This equation can be integrated out to any proper radius $\ell$ to give

$$
R \omega_{+}=-2(M-P)+2 \Gamma_{+},
$$

where

$$
\Gamma_{+}=\int_{0}^{\ell} d \ell F_{+}\left(\omega_{+}, \omega_{-}, \alpha\right)
$$

and 


$$
F_{+}=\frac{1}{8}\left[4+\alpha \omega_{+} \omega_{-}+(1-\alpha) \omega_{+}^{2}\right] .
$$

$\Gamma_{+}$is the natural optical scalar generalization for future trapped surfaces of $\Gamma$ introduced in [2]. In particular, when $K_{a b}=0, \Gamma_{+}=\Gamma$.

Let us assume that there are no future trapped surfaces inside some given radius. For $\alpha \geq 1$, we then have the crude bound

$$
F_{+} \leq \frac{1}{8}\left[4+\alpha \omega_{+} \omega_{-}\right] \leq \frac{1}{2}(1+\alpha) .
$$

We can, however, do much better in this range by exploiting the control we possess over $\omega_{+}$ and $\omega_{-}, 0<\omega_{+} \leq 2$ and $\omega_{-} \leq 2$, to bound $\Gamma_{+}$in a more subtle way.

We are interested in finding an upper bound for

$$
F_{+}^{*}=\alpha \omega_{+} \omega_{-}+(1-\alpha) \omega_{+}^{2},
$$

assuming $1 \leq \alpha<\infty, \omega_{-} \leq 2$ and $0<\omega_{+} \leq 2$.

The first thing we observe is that since both $\alpha$ and $\omega_{+}$are positive we maximize $F_{+}^{*}$ by setting $\omega_{-}=2$. Thus we seek an upper bound for

$$
F_{+}^{* *}=2 \alpha \omega_{+}+(1-\alpha) \omega_{+}^{2} .
$$

The value of this quartic is zero when $\omega_{+}=0$ and 4 when $\omega_{+}=2$. The maximum of the quartic occurs at

$$
\omega_{+}=\frac{\alpha}{\alpha-1}
$$

and it equals

$$
F_{+\max }^{* *}=\frac{\alpha^{2}}{\alpha-1}
$$

there. However, it turns out that $\alpha /(\alpha-1)>2$ when $\alpha<2$ so the maximum of $F_{+}^{* *}$ in that range is 4 , independent of $\alpha$. In other words

$$
F_{+}^{*} \leq \begin{cases}4 & 1 \leq \alpha \leq 2 \\ \frac{\alpha^{2}}{\alpha-1} & \alpha>2\end{cases}
$$

Hence we can derive the useful inequality

$$
F_{+} \leq \begin{cases}1 & 1 \leq \alpha \leq 2 \\ 1+\frac{\alpha}{8(\alpha-1)} & \alpha>2,\end{cases}
$$

assuming, of course, $\omega_{-} \leq 2$ and $0<\omega_{+} \leq 2$. This inequality is clearly sharp.

II.3 Sufficiency Condition: $1 \leq \alpha \leq 2$

It is clear, from (31), that if $\alpha$ lies between 1 and 2 (and, let us stress again, need not be constant) then we get that $\Gamma_{+} \leq \ell$. Thus it immediately follows from (22) that if $M-P>\ell$ then the region must contain a future trapped surface. 
II.4 Sufficiency Condition: $1 \leq \alpha<\infty$

It is clear that the inequality no longer holds if $\alpha>2$. We have instead the weaker bound for all $\alpha \geq 1$ :

$$
F \leq 1+H(\alpha)
$$

where

$$
H(\alpha)= \begin{cases}0 & 1 \leq \alpha \leq 2 \\ \frac{1}{8} \frac{\alpha^{2}}{(\alpha-1)}-\frac{1}{2} & \alpha \geq 2\end{cases}
$$

Let $\langle f\rangle$ represent the average of any function $f$ over the domain $[0, \ell]$. We then have

$$
\Gamma_{+} \leq(1+\langle H(\alpha)\rangle) \ell
$$

$H$ will be finite if $\alpha$ is bounded. Asymptotically, however, $H$ grows linearly with $\alpha$. This reflects the fact that, in this limit, the gauge is pathological precisely at a horizon so we would not expect any kind of reasonable estimate.

If $\omega_{+}>0$ on any spherical domain which includes the origin then

$$
M-P<(1+\langle H(\alpha)\rangle) \ell
$$

A constant of proportionality has been introduced which is given by the spatial average of $1+H$ over the domain of $\ell$. If this inequality is violated we must have a future trapped surface in the domain.

The dependence on $\alpha$ is surprisingly weak. Clearly, no extra cost is involved in accomodating a variable $\alpha$. In fact, the inequality does not depend explicitly on the gradient of $\alpha$.

\section{II.5 Sufficiency Condition: $0.5 \leq \alpha<\infty$}

If $0.5 \leq \alpha<1$, the derivation above breaks down. To bound $\omega_{+}$, we will asssume that not only is the interior geometry regular and not contain any future trapped surfaces but, in addition, that it contain no past trapped surfaces. Then Eq.(18) holds. We now have

$$
F \leq \frac{1}{2}\left(1+\alpha+\operatorname{Max}\left(\frac{1-\alpha}{\alpha_{\operatorname{Min}}}, 0\right)\right)
$$

where we exploit the positivity of the quasi-local mass to bound $\omega_{+} \omega_{-} \leq 4$ and (18) to bound $\omega_{+}$. 


\section{APPARENT HORIZONS: WEAK ENERGY}

\section{III.1 Bounds on the Configuration Space in Regions without Trapped Surfaces}

Let now us relax the energy condition to weak energy, $\rho \geq 0$.

First, we will again assume only that the geometry in our region is regular and that $\omega_{+}>0$ there. Without the additional control over $J$, it is not reasonable to expect $K_{R}$ to be bounded in any simple way. In globally regular geometries,

$$
-1 \leq R^{\prime} \leq 1
$$

everywhere [3]. If we do not have global regularity, the lower bound on $R^{\prime}$ can be breached. What we have instead is the weaker bound,

$$
-\operatorname{Max}\left(1,\left|R K_{R}\right|_{\omega_{+}=0}\right) \leq R^{\prime} \leq 1
$$

The upper bound is a topological one analogous to that on $\omega_{+}$and $\omega_{-}$derived above. The lower bound is a consequence of the fact that when $\omega_{+}>0$, then $R^{\prime} \geq-R K_{R}$. Suppose $R^{\prime}<-1$ at some point $\ell_{0}<\ell$. We know that if $R^{\prime}<-1, R^{\prime \prime}<0$ so that it will henceforth become more negative [3]. Thus the mimimum value of $R^{\prime}$ under these circumstances is the horizon value as claimed. This is not a marvellous result — but will be sufficient for our purposes.

If we relax the hypothesis to global regularity we can exploit Eq.(37).

\section{III.2 Sufficiency Condition: $1 \leq \alpha<\infty$}

Suppose that $\rho \geq 0$, and $1 \leq \alpha<\infty$ but is otherwise unspecified. We will show that if

$$
M-P \geq\langle f(\alpha)\rangle \ell
$$

in some region, where

$$
f(\alpha)=1+\frac{1}{2} \frac{(1-\alpha)^{2}}{2 \alpha-1}
$$

the spatial geometry in this region must possess a future trapped surface or a singularity. This exactly reproduces the condition Eq.(11) when $\alpha=2$. However, the minimum of $f(\alpha)$ is assumed when $\alpha=1$ where we reproduce Eq.(10). Curiously, the gauge providing the best bound when we do not assume dominant energy is not maximal slicing. The likely reason for this is that in this gauge, $K_{R}=P / R^{2}$. Here, weak energy does as well as dominant energy.

The original proof in [7,5] for $K=0$ exploited conformal coordinates. Our approach eschews tying ourselves to any particular spatial coordinate. Not only is the end result independent of the spatial coordinate, it is clear that the coordinate invariant approach is not only more transparent but also more efficient. We will work with the metric variables 
$R$ and $K_{R}$ - when dominant energy is relaxed, the advantage we gain from exploiting the optical scalars is lost. When Eq.(5) holds we can rewrite Eq.(11) in the form

$$
4 \pi \rho R^{2}+\left(R R^{\prime}\right)^{\prime}=\frac{1}{2}\left(1+\left(R^{\prime}\right)^{2}\right)+\frac{1-2 \alpha}{2} R^{2} K_{R}^{2} .
$$

We integrate from $\ell=0$ up to the boundary at proper radius $\ell$ :

$$
M+R R^{\prime}=\Gamma+\int_{0}^{\ell} d \ell\left(\frac{1-2 \alpha}{2}\right) R^{2} K_{R}^{2}
$$

where $\Gamma$ is defined by

$$
\Gamma=\frac{1}{2} \int_{0}^{\ell} d \ell\left[1+\left(R^{\prime}\right)^{2}\right]
$$

We now eliminate $R^{\prime}$ in the surface term in favor of the optical scalar $\omega_{+}$and $K_{R}$ using the defining relation (6).

To eliminate the $K_{R}$ dependence on the boundary which comes along with the replacement of $R^{\prime}$ by $\omega_{+}$, we note that we can integrate the momentum constraint, Eq.(2) to obtain (9)

$$
R^{2} K_{R}=P+\int_{0}^{\ell} d \ell(1-\alpha) R R^{\prime} K_{R}
$$

We do not need an explicit solution of the momentum constraint. In fact, the only explicit use we will make of the momentum constraint is Eq. (44) which does not depend on the possible spatial variation of $\alpha$.

Substituting Eq.(6) and (44) into (42) we now obtain

$$
M-P+2 \omega_{+}=\Gamma+\frac{1}{2} \int_{0}^{\ell} d \ell(1-2 \alpha) R^{2} K_{R}^{2}-\int_{0}^{\ell} d \ell(1-\alpha) R R^{\prime} K_{R} .
$$

Let us label the second and third terms on the right hand side, $I_{1}$ and $I_{2}$ respectively. When $\alpha>0.5, I_{1}$ is manifestly negative. As such we could discard it to cast (45) as an inequality. However, it is clear that we can do better by first completing the square in the sum of $I_{1}$ and $I_{2}$ before discarding:

$$
I_{1}+I_{2}=\frac{1}{2} \int_{0}^{\ell} d \ell(1-2 \alpha)\left(R K_{R}-\frac{1-\alpha}{1-2 \alpha} R^{\prime}\right)^{2}+\frac{1}{2} \int_{0}^{\ell} d \ell \frac{(1-\alpha)^{2}}{2 \alpha-1} R^{\prime 2}
$$

Now, if $R^{\prime 2} \leq 1$, we have

$$
I_{1}+I_{2} \leq \frac{1}{2}\left\langle\frac{(1-\alpha)^{2}}{2 \alpha-1}\right\rangle \ell .
$$

In addition, under these conditions, we obtain the upper bound on $\Gamma$,

$$
\Gamma \leq \ell
$$

This is, however, only the case when we assume that the geometry is regular everywhere. Let us suppose that $R^{\prime}=-1$ at some value $\ell_{0}<\ell$. Then, we can decompose the right 
hand side of Eq.(45) into a part coming from the integration over the domain $\left[0, \ell_{0}\right]$ and a remainder from $\left[\ell_{0}, \ell\right]$ : The integrand, $\Phi$, appearing in this latter contribution can be bounded as follows

$$
\Phi \leq\left|R K_{R}\right|_{\omega_{+}=0}(1+|\alpha-1|-\alpha)
$$

which is bounded by zero if $\alpha \geq 1$, so that we can discard it. We conclude that if the region contains no future trapped surfaces and its geometry is regular then

$$
M-P<\left(1+\left\langle\frac{1}{2} \frac{(1-\alpha)^{2}}{2 \alpha-1}\right\rangle\right) \ell .
$$

This completes the proof.

If we extend the range of $\alpha$ to values below +1 , not surprisingly, we need to relax the hypothesis. However we decide to do this, we appear to require control over the form of the exterior geometry. One might try to weaken the hypothesis to permit us to assume that the entire spatial geometry (from its base out to infinity) is free of future trapped surfaces. However, there is no tactical advantage to this - unlike the moment of time symmetry analysis where this does imply that the geometry is non-singular, here a past apparent horizon could turn up in the exterior with a singularity lurking beyond it.

We need to assume that the entire geometry possesses neither future nor past horizons or weaker, is regular not just within the region of interest. The result will be that one cannot conclude that the apparent horizon or singularity lies necessarily within the region when the inequality holds - it could exist beyond it.

Under these conditions, we again obtain Eq.(39) with the same function $f(\alpha)$. We note that $f$ diverges as we approach the minisuperspace lightcone, $\alpha=0.5$ and $\alpha \rightarrow \infty$. This occurs because the discarded negative term blows up at these two values. At the former value, a more ingeniously constructed proof is likely to remove this infinity. At the latter value, however, the divergence is a genuine signal of the breakdown of the gauge.

III.3 Sufficiency Condition: $0.5 \leq \alpha<\infty$

We have encountered genuine obstructions to finding strong sufficient conditions for the existence of future horizons as one approachee the superspace lightcone. Interestingly, one can find a very simple sufficient condition which is valid for the whole range of $\alpha$ 's if we do not distinguish between future and past horizons.

Let us consider a region around the origin, and let us assume that there are no trapped surfaces in this region. We immediately get that in this region we have

$$
0<R^{\prime} \leq 1
$$

just assuming that the weak energy condition, $\rho \geq 0$, holds.

Let us consider Eq.(41) and integrate it out to any $\ell$ in the region with no trapped surfaces. We replace $\left(R^{\prime}\right)^{2}$ by 1 , and using the facts that $R R^{\prime}>0$ and that $(1-2 \alpha) R^{2} K_{R}^{2} \leq 0$ we get that

$$
M<\ell
$$


Therefore we have shown that if the weak energy condition holds and if $M \geq \ell$ then we must have a trapped surface within this sphere. This result is an old moment-of-timesymmetry result in disguise. We do not need to assume absence of trapped surfaces to get the inequality, all we need to assume is the absence of a minimal surface, i.e., $R^{\prime} \geq 0$, to get that $M<\ell$. Therefore we have shown that if $M \geq \ell$ then there must be a minimal surface within this sphere. Of course, a minimal surface must be a future or a past trapped surface.

We can even do better! The term $(1-2 \alpha) R^{2} K_{R}^{2} / 2$ which we threw away can be brought over to the other side and reunited with the $4 \pi \rho R^{2}$ term. This combination is nothing but one quarter of the three scalar curvature, which must be positive by the combination of the requirement that $\alpha \geq 0.5$ and the weak energy condition. Therefore we have recovered the result that if we have a spherical manifold with positive scalar curvature and if the volume integral of the scalar curvature within a sphere is greater than $16 \pi \ell$, where $\ell$ is the proper radius of the sphere, then there must be a minimal surface within this sphere [2].

\section{SPECIAL CASES}

Let us now examine some extreme cases: for simplicity we will only consider dominant energy.

Let us consider the two extreme distributions satisfying the average dominant energy condition everywhere, but only just, so that $P \rightarrow \pm M$ [10]. If $|J|=\rho$, these are respectively the cases of a radially outward and a radially inward moving null fluid. In the case $P \rightarrow$ $M$, Eq.(10) becomes a vacuous statement, not surprisingly, as it is impossible to form an apparent horizon under such circumstances. All we need to do is look at Eq.(17) and see that if $\rho=J$ and if $\omega_{+}$is small then $\left(\omega_{+}\right)^{\prime} \simeq 1 / R>0$. Thus $\omega_{+}$can never pass through zero from above and since it starts off equalling 2 , it must remain positive. In the latter case, we have that if $2 M \geq \ell+\langle H(\alpha)\rangle \ell$, the geometry will possess a future trapped surface. It is twice as easy to form an apparent horizon with an inflowing null fluid as it is with a stationary fluid.

It is also possible to tighten the sufficiency condition in the same way we did in [2] for $K_{a b}=0$ when $\rho^{\prime} \leq 0$ if, in addition, we demand that $J$ has a fixed sign. What we do substitute for $R^{\prime 2}$ in Eq.(48) using the definition of the quasilocal mass. We have

$$
\begin{aligned}
m & =4 \pi \int_{0}^{\ell} d \ell R^{2}\left[\rho R^{\prime}+J R K_{R}\right] \\
& =\frac{4 \pi}{3} \rho R^{3}+4 \pi \int_{0}^{\ell} d \ell R^{2}\left[J R K_{R}-\rho^{\prime} R\right] \\
& \geq \frac{4 \pi}{3} \rho R^{3}+4 \pi \int_{0}^{\ell} d \ell R^{3} J K_{R} \\
& \geq \frac{4 \pi}{3} \rho R^{3} .
\end{aligned}
$$

The inequality on the second last line obtains when $\rho^{\prime} \leq 0$. If $J$ is positive (or negative) everywhere (In the gauges we are considering $K_{R}$ possesses the same sign as $J$ ) we are left with a sum of two positive terms and so the last inequality holds. We now have

$$
R^{\prime 2}=1-2 m / R+R^{2} K_{R}^{2} \leq 1-\frac{4 \pi}{3} \rho R^{2}+R^{2} K_{R}^{2}
$$


so that

$$
\Gamma \leq \ell-\frac{M}{3}+\frac{1}{2} \int_{0}^{\ell} d \ell R^{2} K_{R}^{2}
$$

Let us examine how this modifies Eq.(39). The last term on the right can now be added to the (negative) term of the same form in Eq.(45) before the completion of the square. We get

$$
\frac{4 M}{3}-P<\frac{3+\langle\alpha\rangle}{4} \ell<\langle\alpha\rangle \ell
$$

in the range $\alpha \geq 1$. As before, this is minimized when $\alpha=1$ and when $P=0$ again reproduces the result at a moment of time symmetry. We note, however, that while the left hand side has been improved, the right hand side is weaker.

¿From one point of view, Eq.(57) is not very satisfactory — we have broken the symmetry between $J$ and $\rho$. However, this very asymmetry apparently permits us to write down a non-vacuous sufficiency condition when dominant energy is saturated with $P=M$. Whereas Eq.(50) is vacuous under these conditions, Eq.(57) provides the non-trivial statement: suppose $\rho^{\prime} \leq 0$ and the motion of matter is outward and null, then if

$$
M \geq 3\langle\alpha\rangle \ell
$$

the spatial geometry will possess an apparent horizon. This apparently contradicts the proof of the absence of apparent horizons when one has a null fluid given in the beginning of this section. The resolution of this paradox, as we will demonstrate in the next section, is that Eq.(58) can never be satisfied.

In a forthcoming publication we will examine intrinsic time foliations. What is very encouraging is that essentially the same condition appears despite the very different nature of the foliation [B].

\section{SINGULARITIES}

Whereas the sufficiency conditions for apparent horizon were clearly strengthened by imposing the dominant energy condition, there is no obvious useful way to import dominant energy into the statement of a sufficiency condition for singularities.

We have an obvious generalization of the moment of time symmetry result. We recall that, in general,

$$
M+R R^{\prime}=\Gamma+\int_{0}^{\ell} d \ell\left(\frac{1-2 \alpha}{2}\right) R^{2} K_{R}^{2}
$$

Let us suppose that the geometry is regular everywhere. Let $\rho$ be positive and $\alpha \geq 0.5$. Now $\Gamma$ is bounded by one. Furthermore, $R^{\prime} \leq 1$ so that $R(\ell) \leq \ell$ everywhere on a non-singular geometry and $R^{\prime} \geq-1$. The surface term is therefore bounded from below by $-\ell$. Finally, the second term on the RHS is negative - The $K_{a b}$ dependence is trivially handled. Thus we get

$$
M \leq 2 \ell
$$


The inequality is independent of the value of $\alpha$ so long as it is bounded from below by 0.5 we do as well as we did at a moment of time symmetry. As at a moment of time symmetry, if we place constraints on the sources it is possible to tighten the inequality. We note that when $\rho^{\prime} \leq 0$ and $J$ is positive (or negative) everywhere, we can exploit Eq.(56) to get

$$
M \leq \frac{3}{2} \ell
$$

Thus we see the vacuous nature of Eq.(58).

Unlike the analogous configurations which occur at a moment of time symmetry. which are non - singular, $\rho^{\prime} \leq 0$ is not sufficient to guarantee a non-singular geometry - the geometry can still turn singular if $J$ is large enough. We therefore cannot claim that Eq.(61) represents a universal bound when $\rho^{\prime}<0$ and $J$ is positive (negative).

The singularity condition is not symmetrical in $M$ and $P$. Unlike the apparent horizon conditions, where $P$ shows up in the combination $M \pm P$, it does not arise in a natural way in either Eq.(60) or Eq.(61).

\section{ACKNOWLEDGEMENTS}

We gratefully acknowledge support from CONACyT Grant 211085-5-0118PE to JG and Forbairt Grant SC/96/750 to NÓM 


\section{REFERENCES}

[1] J. Guven and N. Ó Murchadha, Phys Rev D52 758 (1995)

[2] J. Guven and N. Ó Murchadha, Phys Rev D52 776 (1995)

[3] J. Guven and N. Ó Murchadha, Geometric Bounds in Spherically Symmetric General Relativity (1997)

[4] J. Guven and N. Ó Murchadha, Necessary Conditions for Apparent Horizons and Singularities in Spherically Symmetric Initial Data (1997)

[5] P. Bizoń, E. Malec and N. Ó Murchadha Class Quantum Grav 6, 961 (1989)

[6] E. Malec and N. Ó Murchadha Phys. Rev. D50 R6033 (1994)

[7] P. Bizoń, E. Malec and N. Ó Murchadha Phys. Rev. Lett. 61, 1147 (1988)

[8] J. Guven and N. Ó Murchadha, In Preparation.

[9] The privileged role of the gauge with $\alpha=1$ is evident.

[10] We could alternatively consider matter satisfying the weak energy condition locally but satisfying the averaged strong energy condition globally. Local violations of the dominant energy condition are possible. 